\title{
On Consistent Discrimination between Directed and Diffuse Outdoor Illumination
}

\author{
Artur Ottlik and Hans-Hellmut Nagel \\ Institut für Algorithmen und Kognitive Systeme \\ Fakultät für Informatik der Universität Karlsruhe (TH) \\ 76128 Karlsruhe, Germany \\ \{ottlik|nagel\}@iaks. uni-karlsruhe.de
}

\begin{abstract}
This investigation studies the contrast across boundaries of shadows which are cast by moving vehicles or selected stationary objects in a traffic scene onto the road surface. If a sufficient fraction of this subset of hypothetical shadow contours is overlapped by strong edge elements, such a finding is usually taken as a cue that directed sunshine illuminates the traffic scene. Otherwise, diffuse illumination is assumed. Experiments with different illumination states of a traffic scene indicate that such a conclusion appears justified in many cases. It is demonstrated, however, that much more complicated situations may occur which need detailed inferences in order to arrive at a consistent interpretation.
\end{abstract}

\section{Introduction}

Model-based tracking of road vehicles in video sequences can be improved by taking into account the shadow which a vehicle casts onto the road plane. Such an approach requires, however, that it is known whether diffuse or directed daylight illumination prevailed at the time when each image frame had been recorded. Figure 1 illustrates shadow contours, generated by a 3D-model-based tracking approach and overlayed (in green color ${ }^{1}$ ) in addition to the projection of model segments according to the current pose estimate for the depicted vehicle (see, e. g., [1]). The left panel illustrates an example recorded while the sun shone brightly whereas the sky was covered by clouds during recording of the right panel, causing a diffuse illumination of this road scene.

Initial investigations into the exploitation of shadow contours for model-based tracking relied on the user to provide interactively the required knowledge about type and direction of illumination. Frequent evaluations of new and/or long image sequences, especially of outdoor scenes, make it increasingly cumbersome for a user to estimate the prevailing illumination conditions and to provide these estimates interactively to the model-based tracking system. Not surprisingly, attempts have been reported to estimate the illumination conditions automatically, based on the image frame to be evaluated currently [4]. Another recently published approach checks whether or not a shadow contour - cast hypothetically by a tall mast onto the road plane - is covered by edge elements sufficiently well

${ }^{1}$ Difficult to see in printed version; please inspect PDF-version. 

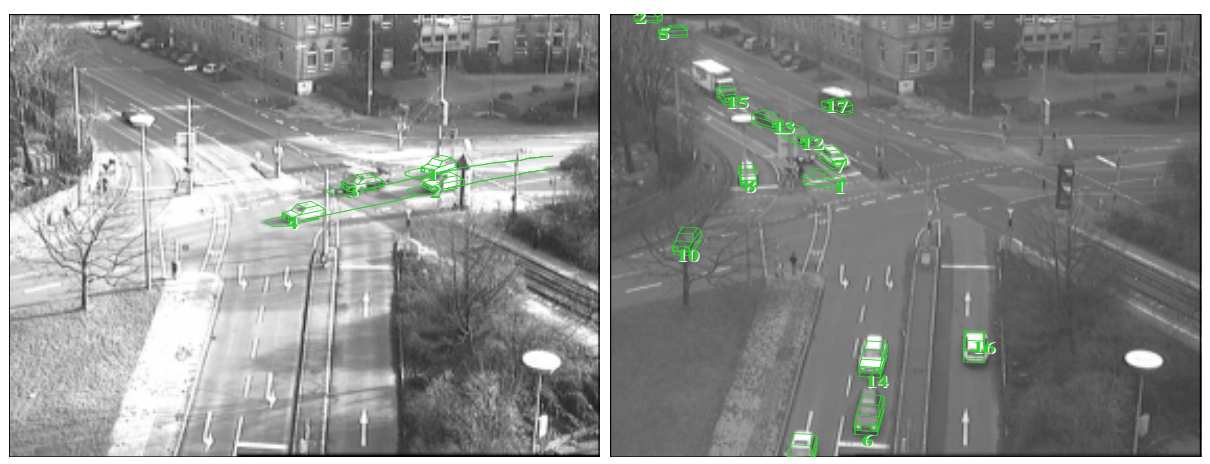

Fig. 1. The left panel shows an image frame recorded while the sun shone brightly whereas the sky was covered by clouds during recording the frame shown in the right panel.

in order to justify the conclusion that the expected shadow is present indeed and thus provides a cue for directed illumination [2].

The latter approach may occasionally result in 'false alarms', for example if edge segments of a moving vehicle passing across the hypothetical shadow contour accidentally align with it. This may generate enough edge elements in the image to let the 'shadow edge coverage' exceed a predefined threshold - see Figure 2. Some temporal continuity requirement may be introduced in order
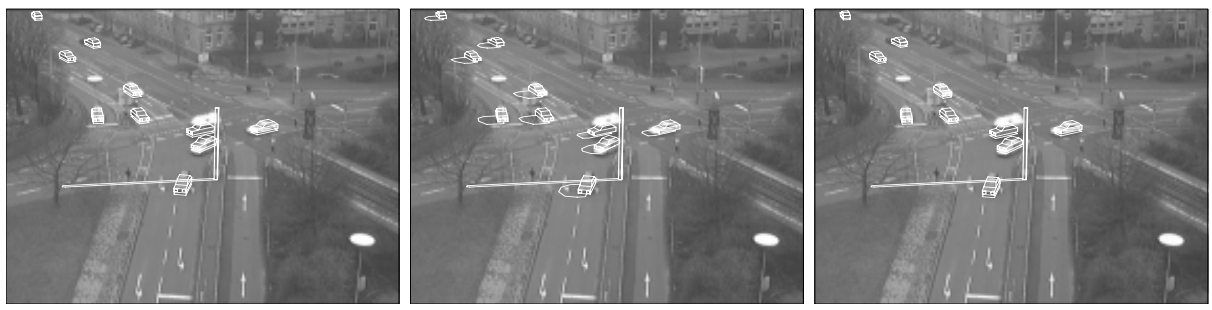

Fig. 2. The left panel shows frame 774 of an image sequence recorded with overcast sky, without extended shadows as expected in such a case. The center panel shows image frame No. 776 where an accidental alignment of a fastback with the hypothetical shadow contour of the tall mast causes a 'false (directed sunshine) alarm'. The tracking process attempts, therefore, to include vehicle shadows into the pose update process. The rightmost panel reproduces frame 778 of this same sequence where this 'false alarm' did no longer occur and matching switched back to the treatment of shadow contours induced by a diffuse illumination.

to avoid such unjustified short switches between illumination states. Experience has shown, however, that this does not solve the problem: on windy days with broken clouds, the illumination state for outdoor scenes may change within about one tenth of a second. Such a period is of the same order as that at- 
tributable to accidental alignments of fast moving image structures with those due to shadow-casting. Search for a consistent interpretation of potential shadow contours within as large a fraction of the field of view as possible promises a more robust approach. Investigations towards such a solution will be reported in the sequel.

\section{A Daylight Illumination Discrimination Approach}

It appears useful to specify as precisely as possible the boundary conditions for the approach to be studied. This will facilitate a later diagnosis whether unsatisfactory results should be attributed to a potential implementation error, to numerical difficulties, to a suboptimal tuning of parameters, or to inappropriate assumptions. In principle, model-based tracking excludes other alternatives as potential explanations for observed failures or insufficiencies. It thus becomes possible to trace down systematically the root causes for unsatisfactory results in order to remove them.

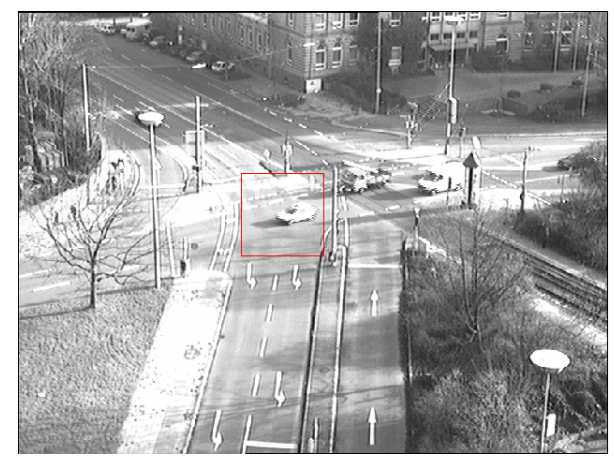

Fig. 3. Frame 135 from the image sequence dtneu04. The red square around the vehicle in the center indicates the cropped image area which is shown in Figure 4.

\subsection{Enumeration of the a priori Knowledge Exploited}

It is assumed that the following knowledge is available to the system:

1. The road traffic scene is recorded by a single, stationary B/W-video camera.

2. All roads within the field of view of the recording camera are situated within the same plane of the depicted scene.

3. Internal parameters of the camera and its pose relative to the road plane.

4. The geographical coordinates (longitude and latitude) of the recorded scene, the date, and the time of day are known for each recorded image frame. Due to the increasing availability of Global Position System (GPS) data, this requirement does no longer constitute a severe bottleneck. Based on these data, the direction can be calculated from which the sun illuminates the scene, provided it is not occluded by clouds. 

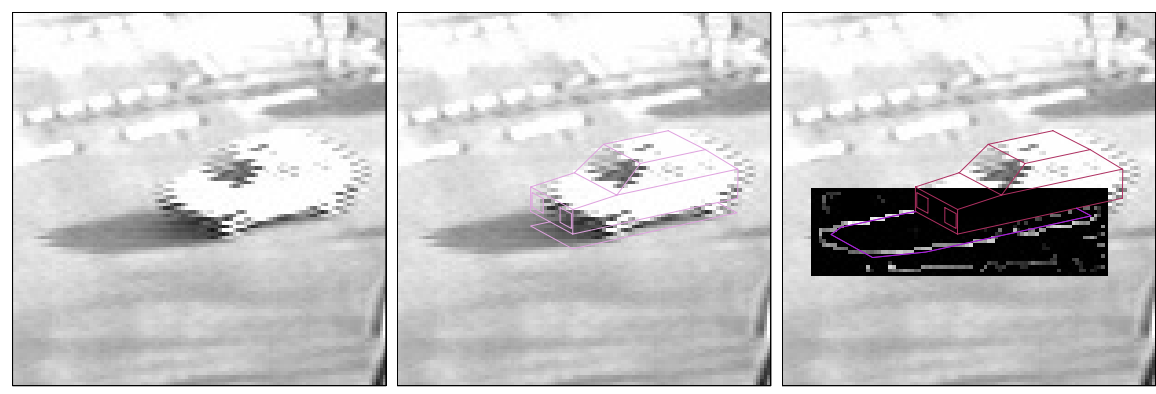

Fig. 4. These panels show a zoom onto a vehicle at frame 135 in the sequence dtneu04. As can be seen in the right panel, many edge elements have been assigned to the shadow segment.
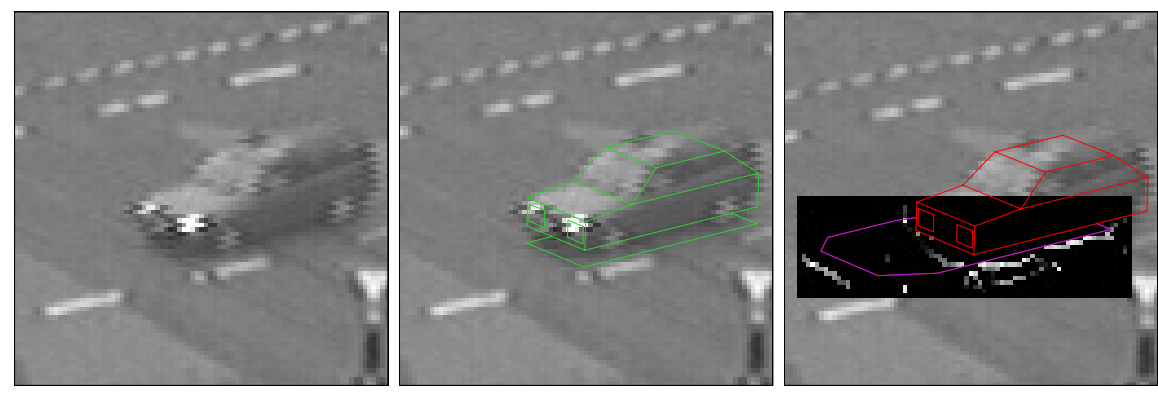

Fig. 5. A zoom onto a vehicle at frame 640 in the sequence dt kont01. The object model is overlayed in the center panel. The right panel illustrates the test for the hypothetical shadow of this vehicle. Bright pixel indicate the (comparatively small number of) edge elements which fit well to the segments of the hypothetical shadow boundary.

5. Boundaries of visible lanes are known in the 3D scene.

6. For each vehicle, its corresponding 3D polyhedral vehicle model is known.

7. Position, height, and diameter of masts carrying traffic lights, traffic indicator boards, or lamps are known in the form of approximating cones and/or polyhedra.

8. 3D polyhedral models are known for other opaque bodies within the field of view of the recording camera to the extent that such bodies may occlude significantly any vehicle to be tracked.

Note that we just exploit knowledge required for model-based tracking; no additional assumptions are introduced. It is assumed further that a procedure is available which can decide whether or not an edge element overlaps a model segment projected into the image plane. Then, either the category diffuse or the category directed has to be assigned to the illumination condition which prevailed while the current image frame had been recorded. 


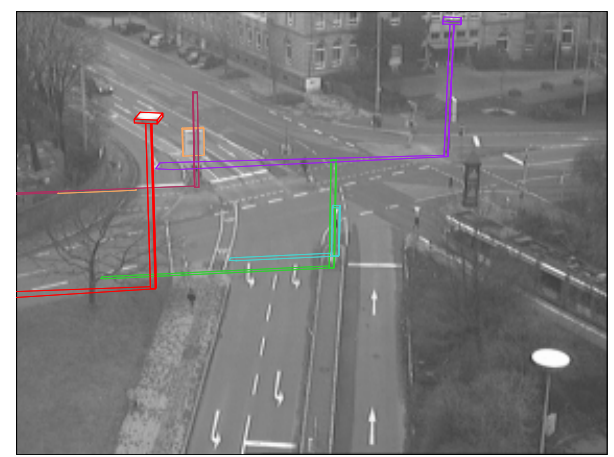

Fig. 6. Contour segments of models of various masts projected into the image plane and overlayed to frame 176 from sequence dt_kont01.

\subsection{Outline of the Estimation Algorithm (Current Version)}

For each image frame, the following steps are executed:

1. Determine the regions corresponding to the image of a road.

2. Use ray-tracing to determine for each road pixel whether it is visible from the camera or not (i. e. occluded by a vehicle or another opaque body).

3. Determine for each road pixel, which is visible to the camera, whether it is visible from the current direction of the sun (illuminated) or not (shadowed).

4. Aggregate hypothetically shadowed road pixels into regions, provided they are visible to the recording camera.

5. Determine the contour of each visible hypothetically shadowed road region.

6. Exclude contour segments where such a hypothetical shadow region touches the silhouette of a shadow-casting body.

7. Determine for each edge element whether it overlaps a visible boundary segment between a shadowed road region and one hypothetically illuminated directly by the sun.

8. Determine a figure-of-merit like the fraction of the total length of visible shadow-road boundaries overlapped by edge elements (shadow/road boundary coverage $(S / R B C))$.

A first approach will compare this $\mathrm{S} / \mathrm{RBC}$ with a threshold in order to distinguish between diffuse $(\mathrm{S} / \mathrm{RBC}$ below threshold) and directed $(\mathrm{S} / \mathrm{RBC}$ at or above threshold) illumination conditions.

\section{Experimental Results}

Figure 3, recorded in bright sunshine, shows an entire frame from which the window presented in Figure 4 has been cropped. The left panel of Figure 4 shows an enlarged window around a fastback. The center panel shows the same window, overlayed by a projection of the polyhedral model for this vehicle at the current best estimate for the vehicle pose. The right panel is similar to the 

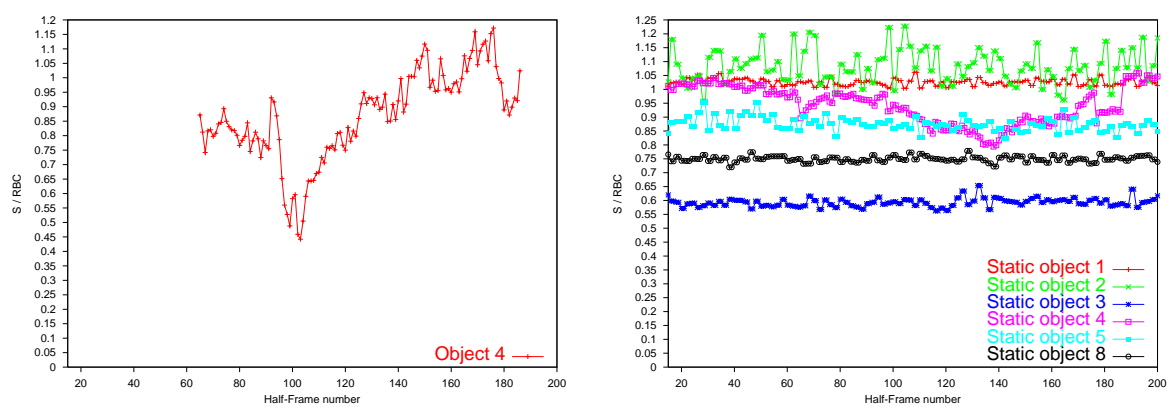

Fig. 7. Left panel: the S/RBC for vehicle object_4 as a function of (half-)framenumber in the sequence dtneu04 recorded in bright sunshine. Right panel: The S/RBC for the masts illustrated in Figure 6, again as a function of the framenumber in the same sequence dtneu04. In both panels, the S/RBC almost always exceeds a value of 0.55 .

center one, but includes the shadow/road boundary. It is clearly discernible that this shadow/road boundary exhibits enough contrast to give rise to numerous strong edge elements. The left panel of Figure 5 shows an enlarged window around a fastback from frame 640 of sequence dt_kont01 recorded at the time of an overcast sky. The right panel corresponds to the right one in Figure 4, including the hypothetical shadow/road boundary expected if the sun would actually shine directly onto this scene: only few edge elements overlap these shadow/road boundaries. In addition to moving objects, the procedure outlined above has been applied to images of tall masts which cast elongated shadows across the scene as illustrated by Figure 6. In case of directed sunshine, results obtained for a vehicle (see left panel of Figure 7) and for several tall masts as illustrated in the right panel of Figure 7 provide evidence as expected: the $\mathrm{S} / \mathrm{RBC}$ exceeds in general 0.55 , in most cases even 0.7. Analogous evidence has been obtained for diffuse illumination as demonstrated in Figure 8, provided accidental alignment of shadow contours with unmodelled scene components are excluded. The dependency of S/RBCs during strong temporal changes of illumination conditions are well illustrated by Figure 9 .

\section{Discussion and Outlook}

It turned out that many component algorithms which have been gradually incorporated into a model-based road vehicle tracking system can be easily combined in order to provide a much more consistent and robust estimate of current illumination conditions. Care has been taken to explicate the conditions which have to be satisfied in order to apply the approach outlined and illustrated in the preceding sections.

Although this approach appears to work fine in case all these seemingly innocuous assumptions are satisfied, it can be upset, e. g., by trees which occlude part of the road with many leafless branches. These branches will cause numerous 

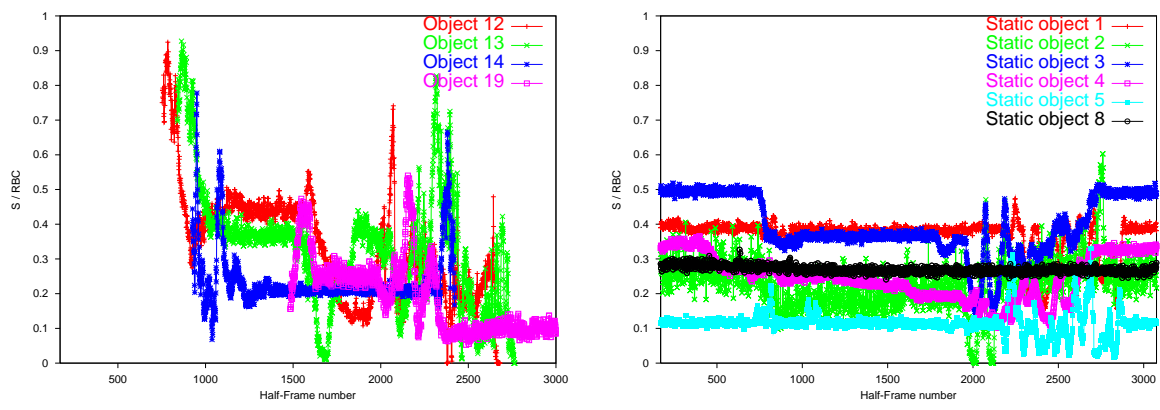

Fig. 8. S/RBCs (left panel: vehicles; right panel: masts) recorded under diffuse illumination in sequence dt kont01. Whereas S/RBCs for the mast shadows consistently remain below $0.6, \mathrm{~S} / \mathrm{RBC}$ for vehicle shadows exceed this value during the beginning and end of this sequence. These 'excess' S/RBC values are due to accidental alignment of shadow contours with unmodelled tree branches and lane markings.

edge elements in practically all orientations with the consequence that enough of them overlap an hypothetical shadow cast onto the road behind these trees. Such areas thus have to be detected and excluded from any attempt to estimate the illumination state.

Another surprising finding has been that even a comparatively small scene like the one illustrated in Figure 3 can be covered by multiple small clouds with fuzzy boundaries such that directed and diffuse illumination coexist in close neighborhood. It thus is no longer possible in general to assume a single daylight illumination state even for such a small scene.

Obviously, a number of additional situations may occur where the list of assumptions enumerated in Section 2.1 is still incomplete. For example, we did not treat the case where large buildings outside the field of view can cast a shadow onto visible parts of the road. In these cases, some vehicles may exhibit strong evidence for directed illumination whereas other ones, driving within areas shadowed by these buildings, give evidence to the contrary. It remains to be seen to which extent such situations can be handled by introducing a corresponding hypothesis, possibly bound to a well delimited part of the scene. In fortunate circumstances, at least a rough estimate of the shadow-casting parts of such buildings may be obtained, in particular if the temporal variation of their shadows with the time of day can be evaluated more closely. Other sources of inconsistent evidence appear possible, for example effects due to a wider range of weather conditions as studied, e. g., in [3]. Nevertheless, the approach described in this contribution appears to provide a good starting point towards gradual improvement of illumination estimation for road traffic scenes in daylight. 

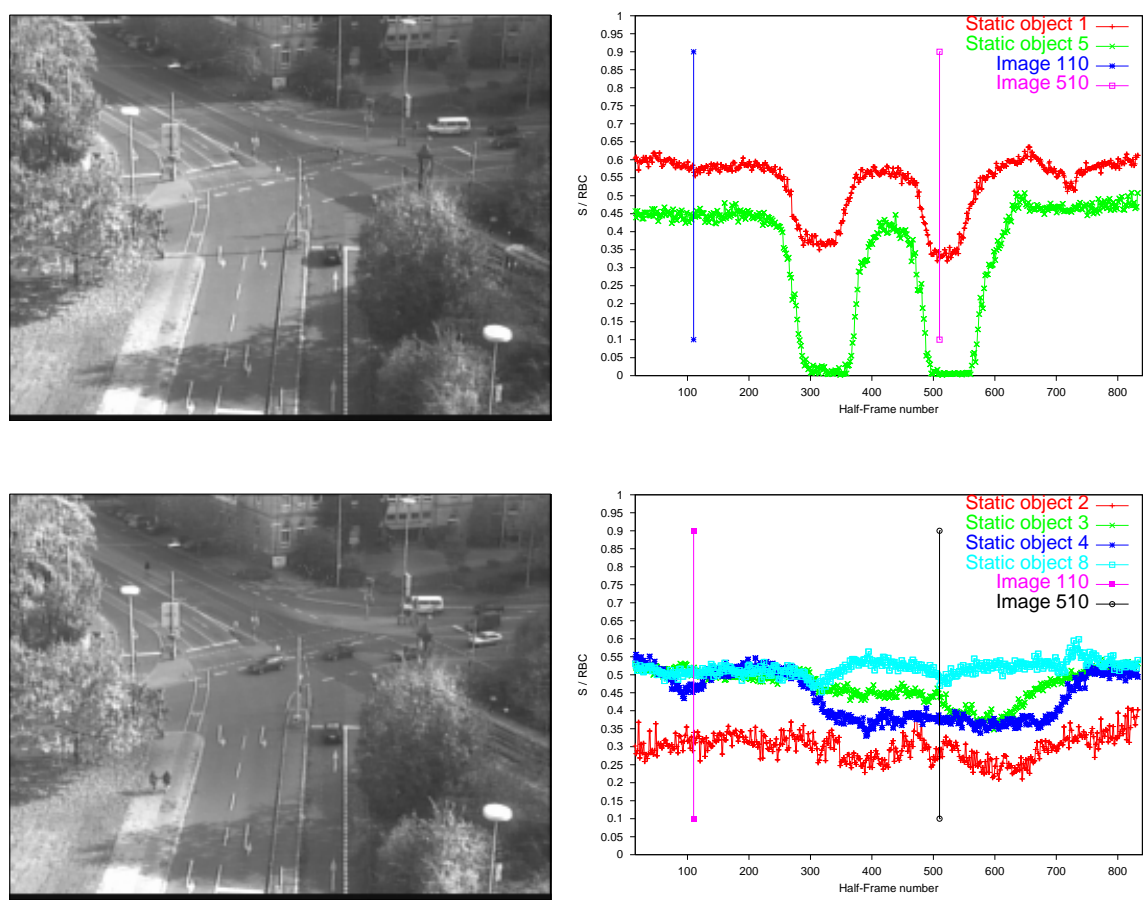

Fig. 9. The 'Shadow/Road Boundary Coverage (S/RBC)' illlustrated during quickly changing illumination conditions. The left panel in the top row shows frame 110 of image sequence dt_kont03a at a time where bright sunshine illuminated most of the depicted scene. The left panel in the bottom row shows frame 510 recorded 8 seconds later when the sky had become partially clouded. The top right panel plots the S/RBC for the two center masts with strong changes of shadow contours on the road. The bottom right panel shows $\mathrm{S} / \mathrm{RBC}$ for other masts where the shadows do not change that markedly during the recording period.

\section{References}

1. D. Koller, K. Daniilidis, and H.-H. Nagel: Model-Based Object Tracking in Monocular Image Sequences of Road Traffic Scenes. International Journal of Computer Vision 10:3 (1993) 257-281.

2. H. Leuck and H.-H. Nagel: Model-Based Initialisation of Vehicle Tracking: Dependency on Illumination. In: Proc. 8th International Conference on Computer Vision (ICCV 2001), 9-12 July 2001, Vancouver/BC, Canada, Vol. I, pp. 309-314.

3. S.G. Narasimhan and S.K. Nayar: Vision and the Atmosphere. International Journal of Computer Vision 48:3 (2002) 233-254.

4. I. Sato, Y. Sato, and K. Ikeuchi: Illumination from Shadows. IEEE Transactions on Pattern Analysis and Machine Intelligence PAMI-25:3 (2003) 290-300. 